\title{
EVALUATING THE GLOBAL THREATS FOR HUMANITY: AN ANALYTIC NETWORK PROCESS MODEL
}

\author{
Mujgan Sagir, Berna Ulutas \\ msagir@,ogu.edu.tr, bhaktan@.ogu.edu.tr \\ Eskisehir Osmangazi University Department of Industrial Engineering \\ 26480 Eskisehir Turkey
}

\begin{abstract}
Throughout human history, there have been many threats to the nations. Global threats are comprised of three main categories, man-made, natural and socio-economic. Man made threats involve every hazard introduced into the natural world that are result of human actions. It includes things such as pollution, wasteful behaviors, wars and terrorist attacks. Natural treats involve natural disasters that can not be avoided towards human actions, as hurricane, earthquake and floods. They are a result of natural cycles. And finally the socio-economic ones involve problems that arise due to societal, political and economic shortcomings as overpopulation, poverty and hunger.

These hazards, coupled with high physical and social vulnerability, may cause unacceptable losses of life, displacement of large number of people, injury, damage to property and devastating economic loss. Earthquakes, landslides, floods, drought, rock falls exacerbate these hazards.

The purpose of this study is to focus on the most important threats that are affecting and/or will be affecting humanity in the future. It is clear that the factors in such phenomenal cases mostly depend on each other. When a disaster occurs the whole system quickly could be turned out to be a much damaged one. An analytic network process model is developed to consider both the dependency and feedback among the criteria. This study could be a motivation point for some other researches in order to evaluate a group of disasters that is especially a threat for a specific nation.
\end{abstract}

Keywords: Global threats, disasters, analytic network process. 